\title{
Properties of Nuclear and Coulomb Breakup of ${ }^{8} \mathrm{~B}$
}

\author{
Kazuyuki OGAta $^{1 *}$, Takuma Matsumoto ${ }^{2 \dagger}$, Yasunori Iseri ${ }^{3}$, and Masanobu YAhiro ${ }^{1}$ \\ ${ }^{1}$ Department of Physics, Kyushu University, 6-10-1 Hakozaki, Fukuoka 812-8581, Japan \\ ${ }^{2}$ RIKEN Nishina Center, 2-1 Hirosawa, Wako 351-0198, Japan \\ ${ }^{3}$ Department of Physics, Chiba-Keizai College, 4-3-30 Todoroki-cho, Inage, Chiba 263-0021, Japan
}

\begin{abstract}
The dependence of breakup cross sections of ${ }^{8} \mathrm{~B}$ at $65 \mathrm{MeV} /$ nucleon on the target mass number $A_{\mathrm{T}}$ is investigated by means of the continuum-discretized coupled-channels method (CDCC) with more reliable distorting potentials than those in the preceding study. The $A_{\mathrm{T}}^{1 / 3}$ scaling law of the nuclear breakup cross section is found to be satisfied only in the middle $A_{\mathrm{T}}$ region of $40 \lesssim A_{\mathrm{T}} \lesssim 150$. The interference between nuclear and Coulomb breakup amplitudes vanishes in very forward angle scattering, independently of the target nucleus. The truncation of the relative energy between the $p$ and ${ }^{7} \mathrm{Be}$ fragments slightly reduces the contribution of nuclear breakup at very forward angles, while the angular region in which the first-order perturbation theory works well does not change essentially.
\end{abstract}

KEYWORDS: CDCC, breakup reaction, ${ }^{8} \mathrm{~B}$, nuclear-Coulomb interference, multistep processes

\section{Introduction}

The properties of unstable nuclei are one of the most important subjects in nuclear physics. The breakup reactions of such short-lived nuclei provide us with much information on their static and dynamical features. The responses to electromagnetic fields of unstable nuclei, which have been intensively studied in particular, are expected to be obtained using breakup reactions induced by a heavy target nucleus such as ${ }^{208} \mathrm{~Pb}$ because of its dominant Coulomb field compared with the nuclear field. Recently, studies of two-neutron halo nuclei, e.g., ${ }^{6} \mathrm{He}$ and ${ }^{11} \mathrm{Li}$, based on this conjecture have been carried out, and $\mathrm{B}(\mathrm{E} 1)$ strengths and spectroscopic factors were evaluated. ${ }^{1,2}$ The extraction of "pure" responses to electromagnetic fields, however, requires an accurate description of the possible nuclear breakup, multistep transitions, and the interference between nuclear and Coulomb breakup amplitudes. The systematic analysis ${ }^{3}$ of the breakup reactions of ${ }^{7} \mathrm{Be},{ }^{8} \mathrm{~B}$, and ${ }^{11} \mathrm{Be}$ with the continuum-discretized coupled-channels method $(\mathrm{CDCC})^{4}$ showed that the nuclear breakup cross section $\sigma_{\mathrm{N}}$ was scaled as $A_{\mathrm{T}}^{1 / 3}$, where $A_{\mathrm{T}}$ is the target mass number. If the scaling is true, one can evaluate $\sigma_{\mathrm{N}}$ induced by a heavy target such as ${ }^{208} \mathrm{~Pb}$, from a measured breakup cross section by a light target such as ${ }^{12} \mathrm{C}$; the latter is expected to contain only nuclear breakup contribution.

\footnotetext{
${ }^{*}$ E-mail: ogata@phys.kyushu-u.ac.jp

${ }^{\dagger}$ Present address: Meme Media Laboratory, Hokkaido University, Sapporo 060-8628, Japan
} 
Thus, there is a possibility that one can remove the "contamination" due to nuclear breakup from the measured breakup cross section by a heavy target, which is generally due to both nuclear and Coulomb breakup. It was also shown, ${ }^{3}$ however, that one could not be free of nuclear-Coulomb (N-C) interference, even if only events corresponding to forward-angle scattering, in which the scattering angle of the center of mass (c.m.) of the projectile is small, are selected. An accurate analysis of the breakup reactions of unstable nuclei with CDCC, therefore, is necessary to draw a quantitative conclusion on $\mathrm{B}(\mathrm{E} 1)$ values and spectroscopic factors. A four-body $\mathrm{CDCC}^{5}$ analysis of breakup reactions of ${ }^{6} \mathrm{He}$ and ${ }^{11} \mathrm{Li}$ is particularly interesting and important.

Before discussing the four-body system, in this study we reinvestigate the breakup reactions of the ${ }^{8} \mathrm{~B}$ nucleus at $65 \mathrm{MeV} /$ nucleon by several target nuclei $\mathrm{A}$, i.e., ${ }^{12} \mathrm{C},{ }^{16} \mathrm{O},{ }^{40} \mathrm{Ca}$, ${ }^{58} \mathrm{Ni},{ }^{90} \mathrm{Zr},{ }^{152} \mathrm{Sm}$, and ${ }^{208} \mathrm{~Pb}$, with $\mathrm{CDCC}$ based on a ${ }^{7} \mathrm{Be}+p+\mathrm{A}$ three-body model. The main purpose of this study is to show some results using more realistic $p$-A and ${ }^{7} \mathrm{Be}-\mathrm{A}$ optical potentials than those in the preceding study. ${ }^{3}$ The $A_{\mathrm{T}}^{1 / 3}$ scaling law of $\sigma_{\mathrm{N}}$ and N-C interference are investigated. As an interesting point not discussed in ref. 3, the dependence of N-C interference on the cut off relative energy between the ${ }^{7} \mathrm{Be}$ and $p$ fragments after breakup is shown here.

This paper is constructed as follows. In $\S 2$, we describe details of numerical inputs of

CDCC. In $\S 3$, the results of the present calculation are shown, and the $A_{\mathrm{T}}^{1 / 3}$ scaling law of $\sigma_{\mathrm{N}}$ and N-C interference are discussed. Finally, we give a summary in $\S 4$.

\section{Numerical Calculation}

We follow the formulation of breakup cross sections with CDCC described in ref. 6 except that we disregard the channel-spin of ${ }^{8} \mathrm{~B}$. We take the ${ }^{8} \mathrm{~B}$ wave function in ref. 7 for the p-wave, with neglecting the spin-orbit potential; the depth of the central part is adjusted to reproduce the proton separation energy $S_{p}=137 \mathrm{keV}$. For the s-wave, the $p-{ }^{7}$ Be potential of Barker $^{8}$ that reproduces the scattering length ${ }^{9}$ for the channel spin $S=2$ component is adopted. As for the d- and f-state potentials, we use the parameters of ref. 10. We take 20, 10, 10, and 5 discretized continuum states for the s-, p-, d-, and f-waves, respectively. The maximum wave number (radius) between $p$ and ${ }^{7} \mathrm{Be}$ is set to be $0.66 \mathrm{fm}^{-1}$ (200 fm). When only nuclear breakup is included, the scattering waves between ${ }^{8} \mathrm{~B}$ and the target are integrated up to $R_{\max }=100 \mathrm{fm}$. The values of $R_{\max }$ for the calculation of nuclear and Coulomb breakup are 210, 210, 250, 330, 450, 670, and $880 \mathrm{fm}$ for the ${ }^{12} \mathrm{C},{ }^{16} \mathrm{O},{ }^{40} \mathrm{Ca},{ }^{58} \mathrm{Ni},{ }^{90} \mathrm{Zr},{ }^{152} \mathrm{Sm}$, and ${ }^{208} \mathrm{~Pb}$ targets, respectively. The maximum orbital angular momentum $L_{\max }$ between ${ }^{8} \mathrm{~B}$ and $A$ is evaluated as $L_{\max }=K_{\mathrm{A}} R_{\max }$, where $K_{\mathrm{A}}$ is the ${ }^{8} \mathrm{~B}$-A relative wave number in the incident channel. For the partial waves with $L>1000$, the eikonal-CDCC method (E-CDCC) ${ }^{6,11}$ is used to obtain the $S$-matrix elements.

We adopt the global optical potential based on Dirac phenomenology (the EDAD1 pa- 
rameter $)^{12}$ for the distorting potential between $p$ and A. As for the ${ }^{7} \mathrm{Be}-\mathrm{A}$ potential $U\left(R_{7}\right)$, we slightly modify the ${ }^{7} \mathrm{Li}$ global optical potential of Cook, ${ }^{13}$ which is appropriate at approximately $10 \mathrm{MeV} /$ nucleon, as

$$
\begin{aligned}
U\left(R_{7}\right)= & -74.03\left\{1+\exp \left[\frac{R_{7}-1.325 A_{\mathrm{T}}^{1 / 3}}{0.853}\right]\right\}^{-1} \\
& -i W\left(A_{\mathrm{T}}\right)\left\{1+\exp \left[\frac{R_{7}-1.640 A_{\mathrm{T}}^{1 / 3}}{0.809}\right]\right\}^{-1}, \\
W\left(A_{\mathrm{T}}\right)= & 31.47-0.160 A_{\mathrm{T}}+0.00045 A_{\mathrm{T}}^{2}
\end{aligned}
$$

Note that the diffuseness parameters are not changed, and the $A_{\mathrm{T}}$ dependence appears only in the radial parameters and the depth of the imaginary part as in the original potential. ${ }^{13}$ This potential is found to reproduce quite well the elastic cross sections of ${ }^{7} \mathrm{Li}^{-12} \mathrm{C}$ at 67.8 $\mathrm{MeV} /$ nucleon ${ }^{14}$ and of ${ }^{7} \mathrm{Be}-{ }^{208} \mathrm{~Pb}$ at $60.8 \mathrm{MeV} /$ nucleon. ${ }^{15}$

\section{Results and Discussion}

First we examine the $A_{\mathrm{T}}^{1 / 3}$ scaling law of $\sigma_{\mathrm{N}}$, which is based on the following two assumptions: $\left.{ }^{3} 1\right)$ the partial breakup cross section $\sigma_{\mathrm{N}}(L)$ is localized around the grazing orbital angular momentum, i.e., $L_{\mathrm{g}} \equiv K_{\mathrm{A}}\left(R_{\mathrm{P}}+R_{\mathrm{T}}\right)$ with $R_{\mathrm{P}}\left(R_{\mathrm{T}}\right)$ being the radius of the projectile (target); and 2) the transmission coefficient at $L_{\mathrm{g}}, T_{L_{\mathrm{g}}}$, is independent of the target nuclei. In Fig. 1 we show $\sigma_{\mathrm{N}}(L)$ for the seven target nuclei calculated with CDCC, with neglecting

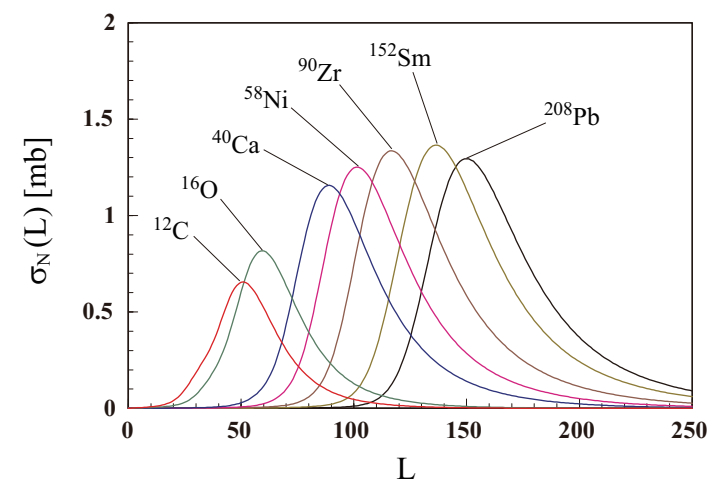

Fig. 1. (Color online) The partial cross sections for nuclear breakup of ${ }^{8} \mathrm{~B}$ by the seven target nuclei concerned.

Coulomb breakup. The values of $L_{\mathrm{g}} / K_{\mathrm{A}}$ and

$$
T_{L_{\mathrm{g}}}=\frac{\sigma_{\mathrm{N}}\left(L_{\mathrm{g}}\right) K_{\mathrm{A}}^{2}}{\pi\left(2 L_{\mathrm{g}}+1\right)}
$$

extracted are shown in the second and fourth rows in Table I, respectively. One sees that $L_{\mathrm{g}} / K_{\mathrm{A}}$ is scaled as $A_{\mathrm{T}}^{1 / 3}$, i.e., $1.4 A_{\mathrm{T}}^{1 / 3}+2.7$ (the third row) reproduces $L_{\mathrm{g}} / K_{\mathrm{A}}$ very well. This 
Table I. $\quad L_{\mathrm{g}} / K_{\mathrm{A}}$ and $T_{L_{\mathrm{g}}}$ extracted from partial cross sections shown in Fig. 1. See text for details.

\begin{tabular}{cccccccc}
\hline$A$ & ${ }^{12} \mathrm{C}$ & ${ }^{16} \mathrm{O}$ & ${ }^{40} \mathrm{Ca}$ & ${ }^{58} \mathrm{Ni}$ & ${ }^{90} \mathrm{Zr}$ & ${ }^{152} \mathrm{Sm}$ & ${ }^{208} \mathrm{~Pb}$ \\
\hline$L_{\mathrm{g}} / K_{\mathrm{A}}[\mathrm{fm}]$ & 6.02 & 6.27 & 7.54 & 8.15 & 9.00 & 10.1 & 11.0 \\
$1.4 A_{\mathrm{T}}^{1 / 3}+2.7[\mathrm{fm}]$ & 5.91 & 6.23 & 7.49 & 8.12 & 8.97 & 10.2 & 11.0 \\
$T_{L_{\mathrm{g}}}$ & 0.14 & 0.19 & 0.29 & 0.30 & 0.31 & 0.28 & 0.25 \\
\hline
\end{tabular}

fact justifies the interpretation of the extracted $L_{\mathrm{g}} / K_{\mathrm{A}}$ as $R_{\mathrm{P}}+R_{\mathrm{T}}$. Namely, the assumption 1) above is shown to be valid. The corresponding $T_{L_{\mathrm{g}}}$, however, shows a clear target dependence. In the middle $A_{\mathrm{T}}$ region of $40 \leq A_{\mathrm{T}} \leq 152, T_{L_{\mathrm{g}}}$ is almost constant, while $T_{L_{\mathrm{g}}}$ significantly decreases for $A_{\mathrm{T}} \leq 16$ and $A_{\mathrm{T}}=208$. This indicates that, in the reactions with a very light or very heavy target, the nuclear absorption at $R_{\mathrm{P}}+R_{\mathrm{T}}$ due to the imaginary parts of the $p$ - $\mathrm{A}$ and ${ }^{7} \mathrm{Be}-\mathrm{A}$ optical potentials is important compared with that in the reactions with middle-heavy targets.

Thus, the $A_{\mathrm{T}}^{1 / 3}$ scaling law of $\sigma_{\mathrm{N}}$ holds in the $40 \lesssim A_{\mathrm{T}} \lesssim 152$ region. In Fig. 2 we show

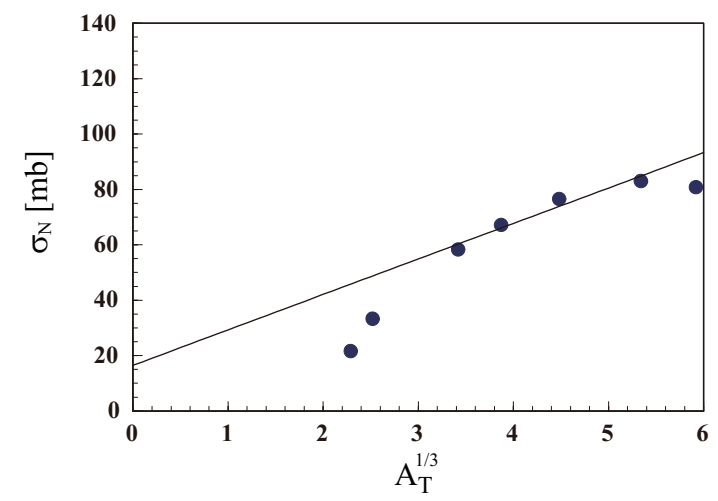

Fig. 2. Nuclear breakup cross section $\sigma_{\mathrm{N}}$ plotted against $A_{\mathrm{T}}^{1 / 3}$. The solid line shows the scaled result $\bar{\sigma}_{\mathrm{N}}\left(A_{\mathrm{T}}^{1 / 3}\right)=12.8 A_{\mathrm{T}}^{1 / 3}+16.5$, obtained by fitting to the $\sigma_{\mathrm{N}}$ for the ${ }^{40} \mathrm{Ca},{ }^{58} \mathrm{Ni},{ }^{90} \mathrm{Zr}$, and ${ }^{152} \mathrm{Sm}$ targets.

the comparison between $\sigma_{\mathrm{N}}$ and the scaling formula

$$
\bar{\sigma}_{\mathrm{N}}\left(A_{\mathrm{T}}^{1 / 3}\right)=12.8 A_{\mathrm{T}}^{1 / 3}+16.5,
$$

which is determined to reproduce the $\sigma_{\mathrm{N}}$ for $A_{\mathrm{T}}=40,58,90$, and 152 . Note that whether the $A_{\mathrm{T}}^{1 / 3}$ scaling law is satisfied or not depends on the properties of the distorting potentials used in the CDCC calculation, which dictates the actual $A_{\mathrm{T}}$ dependence of $T_{L_{\mathrm{g}}}$. In order to perform the systematic analysis of the breakup reactions of ${ }^{11} \mathrm{Be},{ }^{7} \mathrm{Be},{ }^{6} \mathrm{He},{ }^{11} \mathrm{Li}$, and others with threebody or four-body CDCC, therefore, reliable optical potentials between the constituents of the projectile and the targets are necessary. 
Next, we show in Fig. 3 the deviation (relative difference) of the breakup cross section $\sigma_{\mathrm{C}}^{\text {pert }}$, calculated with first-order perturbative CDCC, from the cross section $\sigma_{\mathrm{NC}}^{\text {full }}$ calculated with full CDCC. In the former, one-step transitions due to monopole and dipole Coulomb interactions are only included, while in the latter all the processes due to nuclear and Coulomb interactions are included. The cross sections are integrated over the relative energy $\epsilon$ between the $p$ and ${ }^{7} \mathrm{Be}$ fragments up to $10 \mathrm{MeV}$. The solid, dashed, dotted, and dash-dotted lines

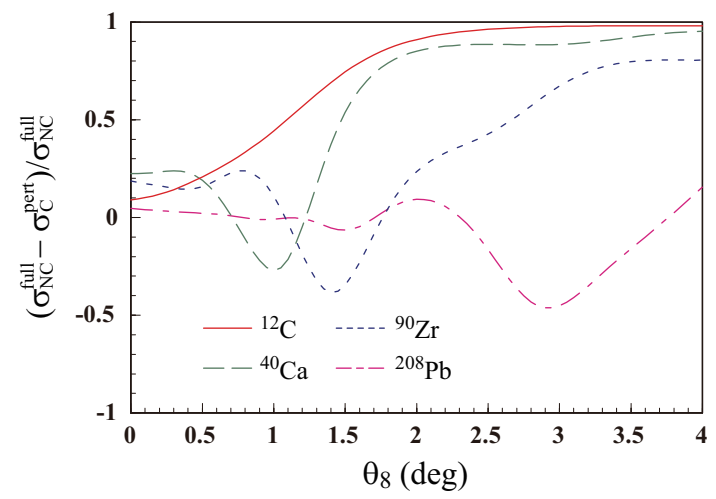

Fig. 3. (Color online) Relative difference between the breakup cross sections calculated with full CDCC and first-order perturbative CDCC, as a function of $\theta_{8}$. The solid, dashed, dotted, and dash-dotted lines correspond to the breakup by ${ }^{12} \mathrm{C},{ }^{40} \mathrm{Ca},{ }^{90} \mathrm{Zr}$, and ${ }^{152} \mathrm{Sm}$, respectively.

in Fig. 3 indicate the results for the ${ }^{12} \mathrm{C},{ }^{40} \mathrm{Ca},{ }^{90} \mathrm{Zr}$, and ${ }^{208} \mathrm{~Pb}$ targets, respectively. The horizontal axis is the c.m. scattering angle $\theta_{8}$ of ${ }^{8} \mathrm{~B}$. As expected, the difference is very large except at forward angles because of the dominant contribution of nuclear breakup. It should be noted, however, that even at very forward angles $\theta_{8} \sim 0^{\circ}$ the difference is somewhat large, i.e., about $10-20 \%$, for the breakup by ${ }^{12} \mathrm{C},{ }^{40} \mathrm{Ca}$, and ${ }^{90} \mathrm{Zr}$ targets. On the other hand, for the breakup by ${ }^{208} \mathrm{~Pb}$, the difference is less than $10 \%$ for $\theta_{8} \leq 2.4^{\circ}$, which justifies in part the use of the first-order perturbation theory to extract "pure" responses of ${ }^{8} \mathrm{~B}$ to electromagnetic fields in this reaction. The possible $10 \%$ error of such simplified analysis, however, should be noted.

Figure 4 shows the relative difference between $\sigma_{\mathrm{NC}}^{\text {full }}$ and $\sigma_{\mathrm{C}}^{\text {pert }}+\sigma_{\mathrm{N}}$. It is found that multistep processes due to Coulomb breakup are negligible in the present case. Thus, $\sigma_{\mathrm{NC}}^{\text {full }}-\left(\sigma_{\mathrm{C}}^{\text {pert }}+\sigma_{\mathrm{N}}\right)$ shows the importance of $\mathrm{N}-\mathrm{C}$ interference. One sees from Fig. 4 that, except for ${ }^{208} \mathrm{~Pb}$, the interference is crucially important for $1.0^{\circ} \lesssim \theta_{8} \lesssim 2.0^{\circ}$. This difference is closely related to the value shown in Fig. 3 in ref. 3, in which it was concluded that the contribution of the interference tended to vanish only for ${ }^{8} \mathrm{~B}$ breakup by ${ }^{208} \mathrm{~Pb}$. Our present calculation shows, however, that the contribution of $\mathrm{N}-\mathrm{C}$ interference almost vanishes at $\theta_{8} \sim 0^{\circ}$ for all target nuclei. Table II shows the maximum scattering angle $\theta_{\text {cr }}$ of the region in which the contribution 


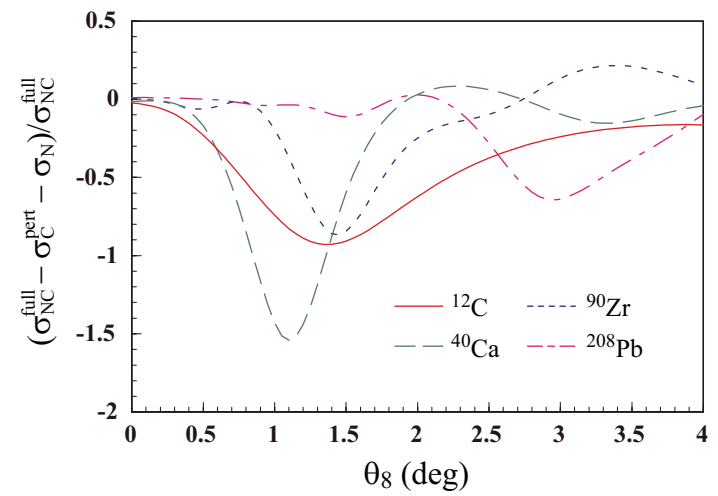

Fig. 4. (Color online) Same as in Fig. 3 but for the relative difference between the breakup cross section calculated with full CDCC and that with first-order perturbative CDCC added by the nuclear breakup cross section.

Table II. Values of $\theta_{\text {cr }}$ and $b_{\text {cr. }}$. See text for details.

\begin{tabular}{cccccccc}
\hline$A$ & ${ }^{12} \mathrm{C}$ & ${ }^{16} \mathrm{O}$ & ${ }^{40} \mathrm{Ca}$ & ${ }^{58} \mathrm{Ni}$ & ${ }^{90} \mathrm{Zr}$ & ${ }^{152} \mathrm{Sm}$ & ${ }^{208} \mathrm{~Pb}$ \\
\hline$\theta_{\text {cr }}[\mathrm{deg}]$ & 0.30 & 0.30 & 0.40 & 0.60 & 0.90 & 1.65 & 2.30 \\
$b_{\text {cr }}[\mathrm{fm}]$ & 26.5 & 31.8 & 47.6 & 42.1 & 38.4 & 31.4 & 29.4 \\
\hline
\end{tabular}

of $\mathrm{N}-\mathrm{C}$ interference is less than $10 \%$, together with the corresponding impact parameters $b_{\mathrm{cr}}$, for the seven reactions concerned.

The $\mathrm{N}-\mathrm{C}$ vanishment at $\theta_{8} \sim 0^{\circ}$ can be explained as follows. In the upper panel of Fig. 5, we show the s- (solid line), p- (dashed line), d- (dotted line), and f-state (dash-dotted line) breakup components of the nuclear breakup cross section of ${ }^{8} \mathrm{~B}$ by ${ }^{208} \mathrm{~Pb}$. One sees that the p-state breakup is dominant at very forward angles. This is because the monopole nuclear coupling potentials are significantly larger than those with other multipolarities, and multistep excitations are negligible in this angular region. On the other hand, dipole transitions are dominant at forward angles in Coulomb breakup. Consequently, as we show in the lower panel, s- and d-wave breakup cross sections are dominant in this case. These features also turn out to be the case for breakup reactions by the other six target nuclei. Thus, the final states of ${ }^{8} \mathrm{~B}$ corresponding to the nuclear breakup and Coulomb breakup are different from each other, with respect to the orbital angular momentum between the ${ }^{7} \mathrm{Be}$ and $p$ fragments. This is the reason for the vanishment of the $\mathrm{N}-\mathrm{C}$ interference at $\theta_{8} \sim 0^{\circ}$. Note that the dominance of the monopole coupling potentials due to nuclear breakup depends on the $p$ $\mathrm{A}$ and ${ }^{7} \mathrm{Be}-\mathrm{A}$ potentials. An accurate evaluation of the distorting potentials used in $\mathrm{CDCC}$ calculation is, again, of crucial importance.

It is sometimes conjectured that the contribution of nuclear breakup can be quenched if 

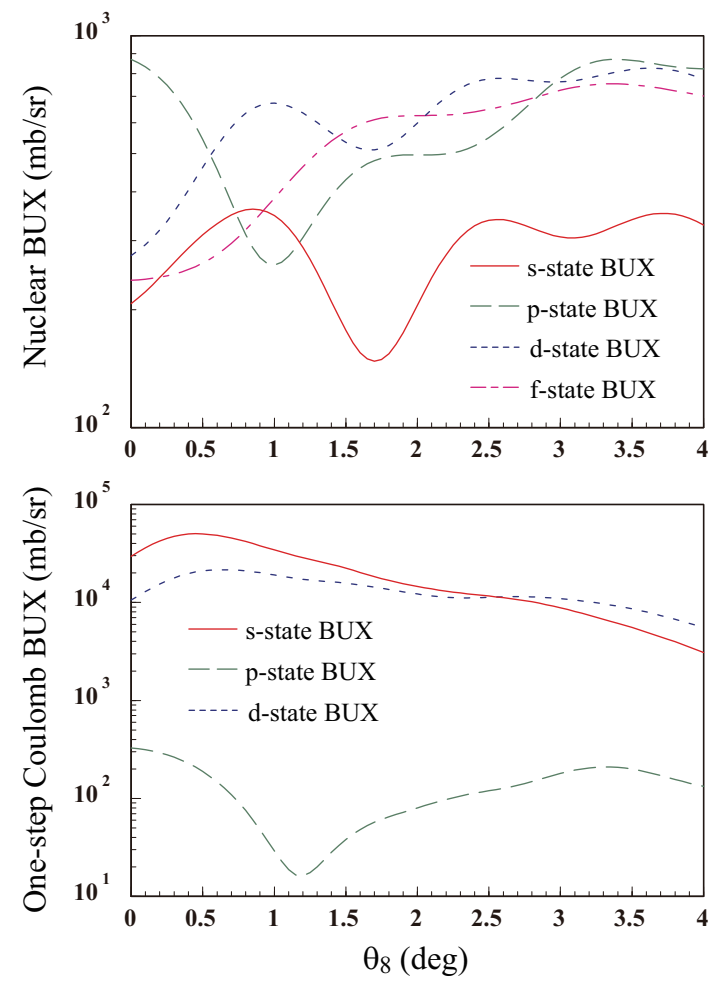

Fig. 5. (Color online) In the upper panel, the nuclear breakup cross section (BUX) by ${ }^{208} \mathrm{~Pb}$ is decomposed into s- (solid line), p- (dashed line), d- (dotted line), and f-state (dash-dotted line) components. The lower panel corresponds to the one-step Coulomb breakup cross section calculated by first-order perturbative CDCC.

the relative energy $\epsilon$ of the fragments is truncated at a certain small value $\epsilon_{0}$. We show in Fig. 6 the relative difference between the breakup cross sections calculated with full CDCC and first-order perturbative CDCC. The thick (thin) lines show the results for the ${ }^{12} \mathrm{C}\left({ }^{208} \mathrm{~Pb}\right)$ target with the truncation at $\epsilon_{0}=0.5$ (solid lines), 1 (dashed lines), and $2 \mathrm{MeV}$ (dotted lines). The results with no truncation are shown by dash-dotted lines for comparison. One sees that the truncation of $\epsilon$ slightly reduces the relative difference at $\theta_{8} \sim 0^{\circ}$. For $\theta_{8} \gtrsim 0.5^{\circ}\left(2.0^{\circ}\right)$ for ${ }^{12} \mathrm{C}\left({ }^{208} \mathrm{~Pb}\right)$, however, the difference is still very large, which indicates that even if truncation of $\epsilon$ is carried out, the first-order perturbative calculation will bring about serious errors in analysis of the breakup reactions in this angular region, and hence in the values of the extracted B(E1) and spectroscopic factor.

\section{Summary}

We reinvestigate the nuclear and Coulomb breakup properties of ${ }^{8} \mathrm{~B}$ at $65 \mathrm{MeV} /$ nucleon by the continuum-discretized coupled-channels method (CDCC) with more reliable $p$-target and ${ }^{7}$ Be-target optical potentials than in the foregoing work. ${ }^{3}$ The $A_{\mathrm{T}}^{1 / 3}$ scaling law of the nuclear breakup cross section $\sigma_{\mathrm{N}}$, with $A_{\mathrm{T}}$ as the target mass number, is found to be satisfied only in 


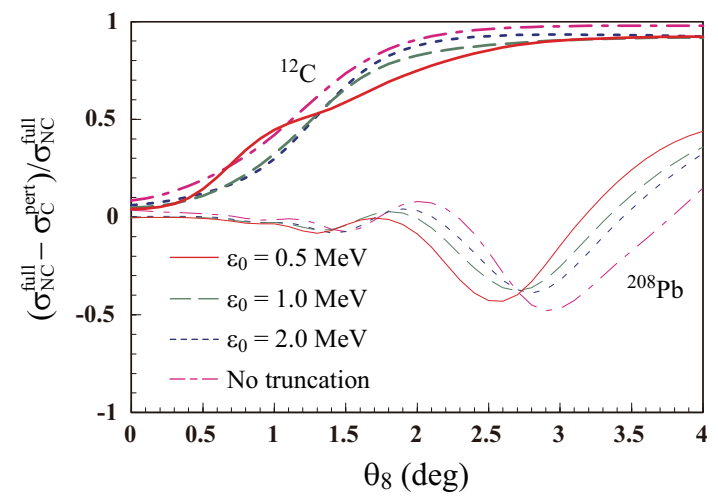

Fig. 6. (Color online) Relative error of the breakup cross section calculated with first-order perturbative CDCC from that with full CDCC. The solid, dashed, and dotted lines correspond to the results with truncation of $\epsilon$ at $0.5,1.0$, and $2.0 \mathrm{MeV}$, respectively. The results with no truncation are shown by the dash-dotted lines. The thick (thin) lines correspond to the breakup by ${ }^{12} \mathrm{C}$ $\left({ }^{208} \mathrm{~Pb}\right)$.

the middle-mass region, i.e., $40 \lesssim A_{\mathrm{T}} \lesssim 152$. The interference between nuclear and Coulomb breakup amplitudes, that is, $\mathrm{N}-\mathrm{C}$ interference, is very important even at forward angles, $1^{\circ} \lesssim \theta_{8} \lesssim 2^{\circ}$, where $\theta_{8}$ is the scattering angle of the center of mass of ${ }^{8} \mathrm{~B}$. For the breakup by ${ }^{208} \mathrm{~Pb}$, it is found that contributions of $\sigma_{\mathrm{N}}$ and $\mathrm{N}-\mathrm{C}$ interference are not so important, i.e., less than $10 \%$, for $\theta_{8} \lesssim 2.4^{\circ}$. The present calculation shows that $\mathrm{N}-\mathrm{C}$ interference indeed tends to vanish at $\theta_{8} \sim 0^{\circ}$, independently of the target nucleus, in contrast to the conclusion in ref. 3. Another clarified fact is that the truncation of the relative energy between the $p$ and ${ }^{7} \mathrm{Be}$ fragments on the measured breakup cross sections quenches the error of the first-order perturbation theory at $\theta_{8} \sim 0^{\circ}$ but is not helpful at all for $\theta_{8} \gtrsim 0.5^{\circ}\left(2.0^{\circ}\right)$ in the breakup by ${ }^{12} \mathrm{C}\left({ }^{208} \mathrm{~Pb}\right)$. One may conclude from the present study that the $\mathrm{B}(\mathrm{E} 1)$ strength of ${ }^{8} \mathrm{~B}$ can be extracted with quite a small error of about $10 \%$ from the analysis of the breakup cross section by ${ }^{208} \mathrm{~Pb}$ for $\theta_{8} \lesssim 2.4^{\circ}$ using the first-order perturbation theory. Nevertheless, an assessment of the error of the perturbation theory is extremely important because it indeed depends on the reaction systems and incident energies. Our final remark is that all the results obtained in the present work reflect the properties of the optical potentials used in the CDCC calculation. For systematic analysis of the breakup reactions of ${ }^{11} \mathrm{Be},{ }^{7} \mathrm{Be},{ }^{6} \mathrm{He},{ }^{11} \mathrm{Li}$ etc. with CDCC, an accurate evaluation of the optical potentials concerned is necessary.

KO thanks S. Bishop for providing elastic cross section data for ${ }^{7} \mathrm{Be}^{2}{ }^{208} \mathrm{~Pb}$ scattering at $60.8 \mathrm{MeV} /$ nucleon. The computation was mainly carried out using the computer facilities at the Research Institute for Information Technology, Kyushu University. 


\section{References}

1) T. Aumann, D. Aleksandrov, L. Axelsson, T. Baumann, M. J. G. Borge, L. V. Chulkov, J. Cub, W. Dostal, B. Eberlein, Th. W. Elze, H. Emling, H. Geissel, V. Z. Goldberg, M. Golovkov, A. Grünschloß, M. Hellström, K. Hencken, J. Holeczek, R. Holzmann, B. Jonson, A. A. Korshenninikov, J. V. Kratz, G. Kraus, R. Kulessa, Y. Leifels, A. Leistenschneider, T. Leth, I. Mukha, G. Münzenberg, F. Nickel, T. Nilsson, G. Nyman, B. Petersen, M. Pfützner, A. Richter, K. Riisager, C. Scheidenberger, G. Schrieder, W. Schwab, H. Simon, M. H. Smedberg, M. Steiner, J. Stroth, A. Surowiec, T. Suzuki, O. Tengblad, and M. V. Zhukov: Phys. Rev. C 59 (1999) 1252; J. Wang, A. Galonsky, J. J. Kruse, E. Tryggestad, R. H. White-Stevens, P. D. Zecher, Y. Iwatai, K. Ieki, Á. Horváth, F. Deák, Á. Kiss, Z. Seres, R. E. Warner, H. Schelin: Phys. Rev. C 65 (2002) 034306.

2) T. Nakamura, A. M. Vinodkumar, T. Sugimoto, N. Aoi, H. Baba, D. Bazin, N. Fukuda, T. Gomi, H. Hasegawa, N. Imai, M. Ishihara, T. Kobayashi, Y. Kondo, T. Kubo, M. Miura, T. Motobayashi, H. Otsu, A. Saito, H. Sakurai, S. Shimoura, K. Watanabe, Y. X. Watanabe, T. Yakushiji, Y. Yanagisawa, and K. Yoneda: Phys. Rev. Lett. 96 (2006) 252502 and the references cited therein.

3) M. S. Hussein, R. Lichtenthäler, F. M. Nunes, and I. J. Thompson: Phys. Lett. B640 (2006) 91.

4) M. Kamimura, M. Yahiro, Y. Iseri, Y. Sakuragi, H. Kameyama, and M. Kawai: Prog. Theor. Phys. Suppl. 89 (1986) 1; N. Austern, Y. Iseri, M. Kamimura, M. Kawai, G. Rawitscher, and M. Yahiro: Phys. Rep. 154 (1987) 125.

5) T. Matsumoto, E. Hiyama, K. Ogata, Y. Iseri, M. Kamimura, S. Chiba, and M. Yahiro: Phys. Rev. C 70 (2004) 061601(R); T. Matsumoto, T. Egami, K. Ogata, Y. Iseri, M. Kamimura, and M. Yahiro: Phys. Rev. C 73 (2006) 051602(R).

6) K. Ogata, S. Hashimoto, Y. Iseri, M. Kamimura, and M. Yahiro: Phys. Rev. C 73 (2006) 024605.

7) H. Esbensen and G. F. Bertsch: Nucl. Phys. A600 (1996) 66.

8) F. C. Barker: Aust. J. Phys. 33 (1980) 177.

9) C. Angulo, M. Azzouzb, P. Descouvemont, G. Tabacaru, D. Baye, M. Cogneau, M. Couder, T. Davinson, A. Di Pietro, P. Figuera, M. Gaelens, P. Leleux, M. Loiselet, A. Ninane, F. de Oliveira Santos, R. G. Pizzone, G. Ryckewaert, N. de Séréville, and F. Vanderbist: Nucl. Phys. A716 (2003) 211.

10) S. Typel, H. H. Wolter, and G. Baur: Nucl. Phys. A613 (1997) 147.

11) K. Ogata, M. Yahiro, Y. Iseri, T. Matsumoto, and M. Kamimura: Phys. Rev. C 68 (2003) 064609.

12) S. Hama, B. C. Clark, E. D. Cooper, H. S. Sherif, and R. L. Mercer: Phys. Rev. C 41 (1990) 2737; E. D. Cooper, S. Hama, B. C. Clark, and R. L. Mercer: Phys. Rev. C 47 (1993) 297.

13) J. Cook: Nucl. Phys. A388 (1982) 153.

14) M.D. Cortina-Gil, P. Roussel-Chomaz, N. Alamanos, J. Barrette, W. Mittig, F. S. Dietrich, F. Auger, Y. Blumenfeld, J. M. Casandjian, M. Chartiera, V. Fekou-Youmbi, B. Fernandez, N. Frascaria, A. Gillibert, H. Laurent, A. Lépine-Szily, N. A. Orrg, J. A. Scarpaci, J. L. Sida, and T. Suomijärvi: Phys. Lett. B401 (1997) 9.

15) S. Bishop: private communication. 